\title{
Lineage Reprogramming: A Promising Way to Generate Hepatocytes
}

\author{
Huofu Qian ${ }^{1,2}$, Xiaomei Li ${ }^{1}$ and Bing Yu${ }^{1 *}$ \\ ${ }^{1}$ Department of Cell Biology, Navy Medical University, China \\ ${ }^{2}$ Department of Gastroenterology, The Second People's Hospital of Taizhou, China
}

*Corresponding author: Bing Yu, Department of Cell Biology, Navy Medical University, 800 Xiangyin Road, Shanghai 200433, PR China.

To Cite This Article: Huofu Qian, Xiaomei Li, Bing Yu, Lineage Reprogramming: A Promising Way to Generate Hepatocytes. Am J Biomed Sci \& Res. 2021 - 11(5). AJBSR.MS.ID.001669. DOI: 10.34297/AJBSR.2021.11.001669.

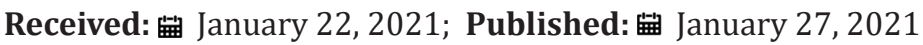

\begin{abstract}
Hepatocyte transplantation is a promising therapy to restore the injured liver function. Currently, donor hepatocytes could be obtained by the isolation from donor liver tissues, the hepatic differentiation of stem cells, including pluripotent stem cells and liver progenitor/stem cells, the direct conversion from other type cells via lineage reprogramming. Here, we review the derived hepatocytes by lineage reprogramming, facilitating the development of efficient reprogramming methods to obtain sufficient functional hepatocytes.
\end{abstract}

Keywords: Liver stem cells; Liver progenitor cells; Lineage reprogramming; Hepatocytes; Differentiation

\section{Introduction}

Hepatocyte transplantation has been shown to be the effective therapy for improving liver functions of emergent injuries to livers and liver-based metabolic disorders [1]. However, the clinical application of hepatocyte transplantation is limited by the shortage of primary hepatocytes which were commonly prepared from donor liver organs. Hepatocytes could also be generated by the hepatic differentiations of pluripotent stem cells, including embryonic stem cells (ESCs) and induced pluripotent stem cells (iPSCs), and liver progenitor/stem cells, which suggesting the newly available cell resources to resolve the shortage of donor hepatocytes [2-4]. However, the potential of tumorigenesis and the immature phenotype of pluripotent stem cell derived hepatocytes limit their applications in cell transplantation [5]. Meanwhile, many studies showed that lineage reprogramming, the direct conversion of one cell type to another cell type by defined factors, enabled more efficiently and safely to obtain hepatocytes when compared with differentiating from pluripotent stem cells [6]. In this minireview, we briefly discuss the strategies for generating hepatocytes through lineage reprogramming.

\section{Hepatocytes Directly Converted from Other Type Cells by Lineage Reprogramming}

Up to now, many tissue functional cells were generated from other type cells by lineage reprogramming [7]. Induced hepatocytes (iHeps) have been manufactured with the different sets of factors in vivo and in vitro by several research groups. In the process of iHeps reprogramming, the cell proliferation and transformation efficiency were often promoted by immortalization of cells, epigenetic modifications, small molecules and the inhibition of cell senescence and death [8-11]. Hence, the characteristics of low conversion efficiency (less than 1\%) and low proliferation may be the major limitations for their translational utilities.

Hepatocytes Generated by Two-Steps: to Derive Liver Progenitor/Stem Cells at First and to Induce them into Hepatocytes Afterward

Liver stem cells possess the capacities of self-renewal and differentiation into mature hepatocytes and cholangiocytes. So, a large amount of mature functional hepatocytes can be obtained from extensively expanded liver stem cells following induced to 
hepatic differentiation. At present, liver stem cells are mainly isolated from liver tissues. Recently, liver progenitor/stem cells were reported to be obtained by de-differentiation of mature hepatoctyes or lineage reprogramming from other type cells. These converted liver progenitor/stem cells can be used as sources to obtain sufficient hepatocytes.

\section{Hepatocytes de-differentiate into hepatic progenitor/ stem cells and then re-differentiate into hepatocytes}

It is well known that primary hepatocytes are difficult to be cultured for a long time in vitro [12]. when maintained in monolayer cultures, primary hepatocytes de-differentiate and rapidly lose hepatocyte-specific functions [13]. Meanwhile, hepatocytes were also found to de-differentiate into proliferative bipotent liver progenitor/stem cells in the chronic injured livers [14,15]. In the past few years, many research groups reprogrammed hepatocytes to de-differentiate into proliferative liver progenitor/stem cells by small molecules in vitro, then this converted hepatic progenitor/ stem cells were induced to re-differentiate into functional hepatocytes [16-18]. By de-differentiation and re-differentiation, sufficient hepatocytes can be obtained from limited donor livers.

\section{Hepatic stem cell generated firstly by lineage reprogramming, then being induced to differentiate into hepatocytes}

Many tissue progenitor/stem cells were generated from other type cells by lineage reprogramming [19]. We firstly directly converted mouse embryonic fibroblasts to induced hepatic stem cells (iHepSCs), with the capacities of both self-renewal and bipotency of differentiation into both hepatocytes and cholangiocytes, by lineage reprogramming with the overexpression of two key transcription factors, Hnf1 $\beta$ and Foxa3 [20]. iHepSCs kept their normal chromosomal numbers and the bipotential differentiation capacity after being extensively expanded (exceeded 50 passages). Importantly, the extensively expanded iHepSCs could be indued to differentiate into functional hepatocytes efficiently in chemical defined medium [21]. Recently, human fibroblast derived hepatocytes were obtained by this two-step procedures [22]. Compared with iHeps, iHepSCs can be expanded in large quantities and then be induced to generate sufficient hepatocytes for cell transplantation, drug screening and liver tissue engineering, etc.

\section{Conclusion}

Compared with iPSCs reprogramming, lineage reprogramming has attracted more and more attention due to its fast reprogramming dynamics, high reprogramming efficiency and low potential tumor risk [6]. Presently, a variety of abundant and easily available cells from different tissues, such as fibroblasts and myofibroblasts, have been successfully reprogrammed into functional hepatocytes or hepatic progenitor/stem cells. Lineage reprogramming could provide sufficient hepatocytes for cell transplantation, drug screen and tissue engineering, etc, so that the source of hepatocytes is no longer only dependent on the donor livers. The strategies for the obtention of hepatocytes by lineage reprogramming were summarized in Figure 1. However, compared with primary hepatocytes, hepatocytes derived from lineage reprogramming are not fully functional and have partial imprints of the original cells. Therefore, the methods of lineage reprogramming still needs to be further improved to obtain reprogrammed hepatocytes with all the functions of primary hepatocytes.

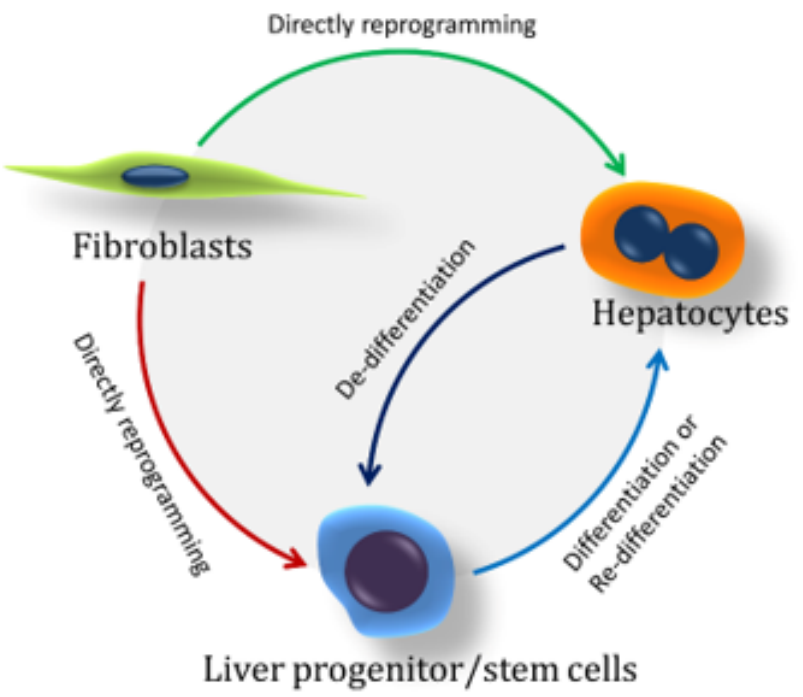

Figure 1: The strategies for the obtention of hepatocytes by lineage reprogramming 


\section{Acknowledgement}

This research was funded by National Natural Science Foundation of China (31771511), and Foundation strengthening program in technical field of China (2019-JCJQ-JJ-068).

\section{Conflict of Interest}

The authors declare no conflict of interests.

\section{References}

1. Bilir BM, Guinette D, Karrer F, Kumpe DA, Krysl J, et al. (2000) Hepatocyte transplantation in acute liver failure. Liver Transplantation 6(1): 32-40.

2. Zhang S, Zhang Y, Chen L, Liu T, Li Y, et al. (2013) Efficient large-scale generation of functional hepatocytes from mouse embryonic stem cells grown in a rotating bioreactor with exogenous growth factors and hormones. Stem Cell Research \& Therapy 4(6): 145.

3. Si-Tayeb K, Noto FK, Nagaoka M, Li J, Battle MA, et al. (2010) Highly efficient generation of human hepatocyte-like cells from induced pluripotent stem cells. Hepatology 51(1): 297-305.

4. Sancho-Bru P, Roelandt P, Narain N, Pauwelyn K, Notelaers T, et al. (2011) Directed differentiation of murine-induced pluripotent stem cells to functional hepatocyte-like cells. Journal of hepatology 54(1): 98-107.

5. Lee AS, Tang C, Rao MS, Weissman IL, Wu JC (2013) Tumorigenicity as a clinical hurdle for pluripotent stem cell therapies. Nature Medicine 19(8): 998-1004.

6. Vierbuchen T, Wernig M (2011) Direct lineage conversions: unnatural but useful? Nature Biotechnology 29(10): 892-907.

7. Kawamata M, Suzuki A (2017) Cell fate modification toward the hepatic lineage by extrinsic factors. Journal of Biochemistry 162(1): 11-16.

8. Huang P, He Z, Ji S, Sun H, Xiang D, et al. (2011) Induction of functional hepatocyte-like cells from mouse fibroblasts by defined factors. Nature 475(7356): 386-389.

9. Huang P, Zhang L, Gao Y, He Z, Yao D, et al. (2014) Direct reprogramming of human fibroblasts to functional and expandable hepatocytes. Cell Stem Cell 14(3): 370-384.

10. Du Y, Wang J, Jia J, Song N, Xiang C, et al. (2014) Human Hepatocytes with Drug Metabolic Function Induced from Fibroblasts by Lineage Reprogramming. Cell Stem Cell 2014 14(3): 394-403.
11. Lim KT, Lee SC, Gao Y, Kim KP, Song G, et al. (2016) Small Molecules Facilitate Single Factor-Mediated Hepatic Reprogramming. Cell reports 15(4): 814-829.

12. Strain AJ (1994) Isolated hepatocytes: use in experimental and clinical hepatology. Gut 35(4): 433-436.

13. Bell CC, Hendriks DF, Moro SM, Ellis E, Walsh J, et al. (2016) Characterization of primary human hepatocyte spheroids as a model system for drug-induced liver injury, liver function and disease. Scientific reports 6: 25187.

14. Tanimizu N, Nishikawa Y, Ichinohe N, Akiyama H, Mitaka T (2014) Sry HMG box protein 9-positive (Sox9+) epithelial cell adhesion moleculenegative (EpCAM-) biphenotypic cells derived from hepatocytes are involved in mouse liver regeneration. The Journal of biological chemistry 289(11): 7589-7598.

15. Tarlow BD, Pelz C, Naugler WE, Wakefield L, Wilson EM, et al. (2014) Bipotential adult liver progenitors are derived from chronically injured mature hepatocytes. Cell Stem Cell 15(5): 605-618.

16. Katsuda T, Kawamata M, Hagiwara K, Takahashi RU, Yamamoto Y, et al. (2017) Conversion of Terminally Committed Hepatocytes to Culturable Bipotent Progenitor Cells with Regenerative Capacity. Cell Stem Cell 20(1): 41-55.

17. Fu GB, Huang WJ, Zeng M, Zhou X, Wu HP, et al. (2019) Expansion and differentiation of human hepatocyte-derived liver progenitor-like cells and their use for the study of hepatotropic pathogens. Cell research 29(1): 8-22.

18. Kim Y, Kang K, Lee SB, Seo D, Yoon S, et al. (2018) Small moleculemediated reprogramming of human hepatocytes into bipotent progenitor cells. Journal of hepatology 70(1): 97-107.

19. Kawamata M, Suzuki A (2017) Cell fate modification toward the hepatic lineage by extrinsic factors. Journal of biochemistry 162(1): 11-16.

20. Yu B, He ZY, You P, Han QW, Xiang D, et al. (2013) Reprogramming Fibroblasts into Bipotential Hepatic Stem Cells by Defined Factors. Cell Stem Cell 13(3): 328-340.

21. Yu B, Li H, Chen J, He Z, Sun H, et al. (2020) Extensively expanded murineinduced hepatic stem cells maintain high-efficient hepatic differentiation potential for repopulation of injured livers. Liver international 40(9): 2293-2304.

22. Xie B, Sun D, Du Y, Jia J, Sun S, et al. (2019) A two-step lineage reprogramming strategy to generate functionally competent human hepatocytes from fibroblasts. Cell research 29(9): 696-710. 